\title{
Spin Currents in Magnetic Nanostructures
}

\author{
V.K. DugaeV ${ }^{a, b}$, P. Bruno ${ }^{c}$ AND J. BARnaŚd,e \\ ${ }^{a}$ Department of Physics, Rzeszów University of Technology \\ al. Powstańców Warszawy 6, 35-959 Rzeszów, Poland \\ ${ }^{b}$ Department of Physics and CFIF, Instituto Superior Técnico \\ Technical University of Lisbon, av. Rovisco Pais, 1049-001 Lisbon, Portugal \\ ${ }^{c}$ European Synchrotron Radiation Facility, BP 220, 38043 Grenoble, France \\ ${ }^{d}$ Department of Physics, Adam Mickiewicz University \\ Umultowska 85, 61-614 Poznań, Poland \\ ${ }^{e}$ Institute of Molecular Physics, Polish Academy of Sciences \\ Smoluchowskiego 17, 60-179 Poznań, Poland
}

\begin{abstract}
We discuss some aspects of the problem of spin currents in magnetic nanostructures. One of them is related to the proper definition of the spin current and is generic for any electronic or magnetic system. Using the standard method related to the symmetry with respect to local spin transformations, we analyze the generation of equilibrium spin currents in inhomogeneous magnetic structures. As an example we consider the generation of persistent spin current in a mesoscopic ring.
\end{abstract}

PACS numbers: 72.25.-b, 73.23.Ra, 75.75.+a

\section{Introduction}

In the past several years considerable efforts have been directed to understanding the peculiarities of spin transport phenomena in magnetic nanostructures. It turned out that the physics of spin-dependent phenomena is surprisingly rich of new effects, which can have potential device applications. The key point of many problems is the spin current: its generation and manipulation by using magnetic fields, bias and gate voltages, light illumination, and so on. Some basic ideas in this field has been suggested by Slonczewski [1] and Berger [2], which resulted in a flow of theoretical and experimental works.

The investigation of spin-dependent transport phenomena in metals or semiconductors requires understanding of peculiarities of the spin-density or spin-current response to an external perturbation. The standard Kubo formalism in 
the frame of a spin-independent response theory is reproduced in many textbooks (see, for example, Refs. [3, 4]). The corresponding generalization to the case of a spin-dependent perturbation in terms of spin currents $[5,6]$ is very nontrivial and still needs further studying [7].

First, the definition of the spin current has been often made only on an intuitive physical ground. On the other hand, it is well known that the charge current is related to the local phase (gauge) transformations and to the conservation of electric charge [8]. For the spin currents, the underlying symmetry is the group of $\mathrm{SU}(2)$ transformations in spin space [6] but this symmetry can be explicitly broken by any spin-depending interactions. Thus, there is a question about using the definition of spin current and its possible non-conservation, i.e., about the existence of continuity equation for spin in the case of scattering from magnetic impurities or spin-orbit interactions, and in the case of spin transport in inhomogeneous magnetic structures. The other question is related to existence of equilibrium spin currents, which do not break time invariance, and are dissipationless.

Recently, several spin-dependent transport effects have attracted much attention, among them the anomalous Hall effect [9, 10] and the spin Hall effect [11-13]. In the first case, the charge Hall current is induced by the electric current flowing in a perpendicular direction, in the absence of external magnetic field. In the second case, the spin Hall current is induced by the perpendicular charge current. Also, the corresponding inverse effects can be possible, like generation of the electric voltage by spin-polarized current [14]. All these phenomena have to be described using a unified formalism, in which the charge and/or spin current are induced by electric or spin perturbations. It should be emphasized that not only the off-diagonal (Hall) components of a tensor determining the response (like $\left.\sigma_{x y}\right)$ are of current interest, but also the diagonal components, connected with the spin conductivity. The corresponding transport effects, described by the diagonal or off-diagonal responses, are related to the charge-to-spin current transformation, which is the key problem of modern spintronics [15].

In this work we also discuss a problem of the spin transport in inhomogeneous magnetic systems related to non-conservation of the spin current and to existence of nondissipative equlibrium spin currents. This problem can be analyzed using some simplified models, which can be realized, for example, in form of magnetic nanorings. The equilibrium spin currents are responsible for the magnetic interactions, and can be identified via the electric polarization appearing in the vicinity of the spin flows.

\section{Spin current in the electronic system}

We start from the standard field theory approach [8] to define the spin current. Our formalism is equivalent to that of Refs. [5, 6]. Let us consider first a nonrelativistic electron gas in the absence of internal magnetization or any spin-dependent interactions. The corresponding Lagrangian has the following form (we put $\hbar=1$ ): 


$$
L=\int \mathrm{d}^{3} \boldsymbol{r} \psi^{\dagger}(\boldsymbol{r}, t)\left(\mathrm{i} \frac{\partial}{\partial t}+\frac{\nabla^{2}}{2 m}-V(\boldsymbol{r})\right) \psi(\boldsymbol{r}, t),
$$

where $\psi(\boldsymbol{r}, t)$ is the two-component spinor field, and $V(\boldsymbol{r})$ is the random potential related to impurities. This Lagrangian is invariant under global spin transformations. Instead, one can perform local spin transformation

$$
\psi(\boldsymbol{r}, t) \rightarrow \exp [-\mathrm{i} g \boldsymbol{n}(\boldsymbol{r}, t) \cdot \boldsymbol{\sigma}] \psi(\boldsymbol{r}, t),
$$

where $\boldsymbol{n}(\boldsymbol{r}, t)$ is a unit vector and $g$ is a coupling constant. The Lagrangian, invariant under such local transformations, is

$$
\begin{aligned}
L= & \int \mathrm{d}^{3} \boldsymbol{r} \psi^{\dagger}(\boldsymbol{r}, t)\left[\mathrm{i}\left(\frac{\partial}{\partial t}-\mathrm{i} A_{0}^{i}(\boldsymbol{r}, t) \sigma^{i}\right)+\frac{1}{2 m}\left(\frac{\partial}{\partial r_{\alpha}}-\mathrm{i} A_{\alpha}^{i}(\boldsymbol{r}, t) \sigma^{i}\right)^{2}\right. \\
& -V(\boldsymbol{r})] \psi(\boldsymbol{r}, t),
\end{aligned}
$$

and it includes gauge fields $A_{0}^{i}(\boldsymbol{r}, t)$ and $A_{\alpha}^{i}(\boldsymbol{r}, t)$. The gauge fields related to transformation (2), are determined by

$$
A_{0}^{i}(\boldsymbol{r}, t)=g \frac{\partial n^{i}(\boldsymbol{r}, t)}{\partial t}, \quad A_{\alpha}^{i}(\boldsymbol{r}, t)=g \frac{\partial n^{i}(\boldsymbol{r}, t)}{\partial r_{\alpha}} .
$$

We define the spin density $S^{i}(\boldsymbol{r}, t)$ and the spin-current density $J_{\alpha}^{i}(\boldsymbol{r}, t)$ operators as the functional derivatives of the Lagrangian

$$
\begin{aligned}
& S^{i}(\boldsymbol{r}, t)=\frac{\delta L}{\delta A_{0}^{i}(\boldsymbol{r}, t)}, \\
& J_{\alpha}^{i}(\boldsymbol{r}, t)=\frac{\delta L}{\delta A_{\alpha}^{i}(\boldsymbol{r}, t)} .
\end{aligned}
$$

Then using Eqs. (3) to (6), we find

$$
\begin{aligned}
& S^{i}(\boldsymbol{r}, t)=\psi^{\dagger}(\boldsymbol{r}, t) \sigma^{i} \psi(\boldsymbol{r}, t) \\
& J_{\alpha}^{i}(\boldsymbol{r}, t)=\frac{1}{2 m} \psi^{\dagger}(\boldsymbol{r}, t)\left[\sigma^{i}\left(-\mathrm{i} \frac{\partial}{\partial r_{\alpha}}-A_{\alpha}^{j}(\boldsymbol{r}, t) \sigma^{j}\right)\right. \\
&\left.+\left(-\mathrm{i} \frac{\partial}{\partial r_{\alpha}}-A_{\alpha}^{j}(\boldsymbol{r}, t) \sigma^{j}\right) \sigma^{i}\right] \psi(\boldsymbol{r}, t) .
\end{aligned}
$$

Let us note that both spin density and spin current density are local in time and space, and both include the gauge potential, which does not vanish in the inhomogeneous magnetic system. The invariance of Lagrangian (3) with respect to small rotations $\boldsymbol{n} \rightarrow \boldsymbol{n}+\delta \boldsymbol{n}(\boldsymbol{r}, t)$ leads to the spin conservation

$$
\frac{\partial S^{i}(\boldsymbol{r}, t)}{\partial t}+\operatorname{div} \boldsymbol{J}^{i}(\boldsymbol{r}, t)=0 .
$$

However, any additional spin-dependent terms in the Hamiltonian, like an exchange field or the spin-orbit (SO) interaction, would destroy the invariance with respect to the spin transformations (2), leading to non-conservation of the spin density. Hence, Eq. (9) holds in the absence of the spin-dependent interactions. 
If we include $\mathrm{SO}$ interaction, it breaks the symmetry, and the spin current is not conserved. However, if the SO interaction is relatively small, we can take it into account as a weak spin relaxation, which can be formally included into right part of Eq. (9) in form of a relaxation term $-\delta S^{i}(\boldsymbol{r}, t) / \tau_{\text {so. }}$. It should be emphasized that the requirement of weak spin relaxation is related to the definition of spin current as conserved quantity, and it gives us a choice with two different possibilities. Either we can try to redefine the spin current (in this case it should be called different) [16], so that the new definition would lead us to a new conservation law, or we still accept standard definition of spin current (5), (6) keeping in mind its non-conservation in spin-relaxation processes or in transfer of torque.

Our consideration of spin currents in the electronic system can be easily generalized to the system described by the Hamiltonian of magnetic interactions. In the following we demonstrate that it leads to appearance of equilibrium spin currents in noncollinear magnetic structures.

\section{Spin current in a ferromagnet with inhomogeneous magnetization}

Let us consider the continuous model of a ferromagnet described by Hamiltonian, which includes the exchange interaction, anisotropy, and the interaction with external magnetic field $\boldsymbol{B}(\boldsymbol{r})$ :

$$
H=\int \mathrm{d}^{3} \boldsymbol{r}\left[\frac{a}{2}\left(\frac{\partial n_{\mu}}{\partial r_{i}}\right)^{2}+\mathcal{F}\{\boldsymbol{n}(\boldsymbol{r})\}-\beta B_{\mu} n_{\mu}\right],
$$

where $\boldsymbol{n}(\boldsymbol{r})$ is the unit vector oriented along the magnetization at the point $\boldsymbol{r}, a$ is the constant of exchange interaction, $\mathcal{F}\{\boldsymbol{n}(\boldsymbol{r})\}$ is a function determining the anisotropy (correspondingly, it includes a certain number of tensors relating the components of vector $\boldsymbol{n}), \beta=g_{\mathrm{L}} \mu_{\mathrm{B}} M_{0}, g_{\mathrm{L}}$ is the Landé factor, $\mu_{\mathrm{B}}$ is the Bohr magneton, and $M_{0}$ is the magnitude of magnetization. Due to the condition $\boldsymbol{n}^{2}(\boldsymbol{r})=1$, this model is constrained and belongs to the class of nonlinear $\sigma$ models [17].

The stationary (saddle point) solutions for the magnetization $\boldsymbol{n}(\boldsymbol{r})$ describing metastable states of the ferromagnet, can be found by minimizing Hamiltonian (10) with the constraint $\boldsymbol{n}^{2}(\boldsymbol{r})=1$. It was shown (see, e.g., Refs. [17, 18]) that such metastable states with inhomogeneous magnetization are related to the topology of ferromagnetic ordering, and they can include hedgehogs, skyrmions, magnetic vortices and other topological objects.

In the magnetic system described by Hamiltonian (10) we can use the same definition of spin current as related to the rotation of vector $\boldsymbol{n}$ in the spin space [19]. In this case, the corresponding transformations are rotations of magnetization vectors belonging to the group $\mathrm{SO}(3)$. Following the idea, we perform a local rotation $\boldsymbol{n}(\boldsymbol{r}) \rightarrow R(\boldsymbol{r}) \boldsymbol{n}(\boldsymbol{r})$ using the orthogonal transformation matrix $\mathrm{R}(\boldsymbol{r})$. By definition, it determines the rotation of local frame in each point of the coordinate space. The general form of transformation is

$$
R(\boldsymbol{r})=\mathrm{e}^{\mathrm{i} \psi(\boldsymbol{r}) J^{z}} \mathrm{e}^{\mathrm{i} \theta(\boldsymbol{r}) J^{y}} \mathrm{e}^{\mathrm{i} \phi(\boldsymbol{r}) J^{z}},
$$


where $\psi, \theta, \phi$ are the Euler angles determining arbitrary rotations of the coordinate frame, and $J^{x}, J^{y}$, and $J^{z}$ are the generators of $3 \mathrm{D}$ rotations around $x, y$ and $z$ axes, respectively.

The Hamiltonian of exchange interaction (the first term in Eq. (10)) in the rotated frame acquires the following form:

$$
H_{\mathrm{ex}}=\frac{a}{2} \int \mathrm{d}^{3} \boldsymbol{r}\left(\frac{\partial n_{\mu}}{\partial r_{i}}-A_{i \mu \nu} n_{\nu}\right)^{2},
$$

where the gauge field $A_{i}(\boldsymbol{r})$ is defined by

$$
A_{i}(\boldsymbol{r})=\left(\frac{\partial}{\partial r_{i}} R(\boldsymbol{r})\right) R^{-1}(\boldsymbol{r}) .
$$

Transformation (11) and the vector components of gauge potential $A_{i}(\boldsymbol{r})$ are $3 \times 3$ matrices acting in the spin space. The matrix $A_{i}(\boldsymbol{r})$ can be presented as

$$
A_{i}(\boldsymbol{r})=i J^{\mu} \mathcal{A}_{i}^{\mu}(\boldsymbol{r}),
$$

where $\mathcal{A}_{i}^{\mu}(\boldsymbol{r})$ belong to the adjoint representation of the group $\mathrm{SO}(3)$.

After transformation to the local frame, the anisotropy described by the second term in the right hand side of (10) results in a function $\tilde{\mathcal{F}}\{\boldsymbol{n}(\boldsymbol{r})\}$ with correspondingly transformed tensor fields. We do not restrict the consideration by any specific form of the anisotropy. The last term in Eq. (10) describing the coupling to external field, has the same form after the transformation, with the vector components in the rotated frame.

The exchange energy term in the Hamiltonian, containing the gauge field associated with the spin rotations, can be written as

$$
H_{\mathrm{ex}}=\frac{a}{2} \int \mathrm{d}^{3} \boldsymbol{r}\left[\left(\frac{\partial}{\partial r_{i}} \delta_{\alpha \beta}-\mathrm{i} \mathcal{A}_{0 i}^{\mu} J_{\mu}^{\alpha \beta}\right) n_{\beta}\right]^{2} .
$$

Here $\mathcal{A}_{0 i}^{\mu}$ is labelled with index 0 to distinguish it from previously considered field $\mathcal{A}_{i}^{\mu}$, which was related to the varying in space orientation of the local frame. The field $\mathcal{A}_{0 i}$ transforms under spin rotations as $\mathcal{A}_{0 i} \rightarrow \mathrm{e}^{\mathrm{i} \boldsymbol{\Omega} \cdot \boldsymbol{J}}\left(\mathcal{A}_{0 i}+\partial \boldsymbol{\Omega} / \partial r_{i}\right)$, where $\boldsymbol{\Omega}(\boldsymbol{r})$ is the angle of spin rotation.

The spin current in the magnetic system is

$$
j_{i}^{\mu}=\gamma \frac{\delta H}{\delta \mathcal{A}_{0 i}^{\mu}},
$$

and we find

$$
j_{i}^{\mu}=-\mathrm{i} c_{s} J_{\mu}^{\alpha \beta} n_{\beta}\left(\frac{\partial}{\partial r_{i}} \delta_{\alpha \gamma}-\mathrm{i} \mathcal{A}_{0 i}^{\delta} J_{\delta}^{\alpha \gamma}\right) n_{\gamma}
$$

where $c_{s}=\gamma a M_{0}$, amd $\gamma$ is the gyromagnetic ratio. The spin current (17) is gauge invariant. We can fix the gauge by taking $\mathcal{A}_{0 i}^{\mu}=0$ since we introduced the potential $\mathcal{A}_{0 i}^{\mu}$ as an auxiliary field to define the spin current. Then, using the relation $\mathrm{i} J_{\mu}^{\nu \lambda}=\epsilon_{\mu \nu \lambda}$, where $\epsilon_{\mu \nu \lambda}$ is the unit antisymmetric tensor, we obtain 


$$
j_{i}^{\mu}=c_{s} \epsilon_{\mu \nu \lambda} n_{\nu} \frac{\partial n_{\lambda}}{\partial r_{i}} .
$$

As follows from Eq. (18), the spin current is nonzero in noncollinear ferromagnets. In particular, it is nonzero in a metastable state of the ferromagnet with topological excitations [18].

Now we calculate the spin current in a thin mesoscopic ring. It is the case when, due to the magnetic anisotropy, the magnetization is noncollinear, and it usually forms a magnetization vortex. For the ring geometry, we use cylindric coordinates $(\rho, \varphi, z)$ of the point on the ring, and assume that the vector $\boldsymbol{n}$ does not depend on $\rho$ and $z$. Then the Hamiltonian $H_{\mathrm{ex}}$ can be written as

$$
H_{\mathrm{ex}}=\frac{a \zeta_{0}}{2 R} \int_{0}^{2 \pi} \mathrm{d} \varphi\left(\frac{\partial n_{\mu}}{\partial \varphi}+\frac{1}{2 \pi} \epsilon_{\mu \lambda \nu} \Phi^{\lambda} n_{\nu}\right)^{2}
$$

where $R$ and $\zeta_{0}$ are the radius and cross-section of the ring, respectively, $\Phi_{0}^{\lambda} \equiv$ $L \mathcal{A}_{0 \varphi}^{\lambda}$, and $L=2 \pi R$. We take $\mathcal{A}_{0 \varphi}^{\mu}$ constant along the ring. Then $\Phi_{0}^{\mu}$ is the flux of the $\mu$-component of fictitious gauge field $\mathcal{A}_{{ }^{\prime}}^{\mu}$ through the ring. The spin current in the ring is

$$
j_{\varphi}^{\mu}=c_{s} \frac{\delta H}{\delta \Phi_{0}^{\mu}} .
$$

Using Hamiltonian (10) and taking the auxiliary field $\Phi_{0}^{\mu}=0$ we find the components of spin current flowing along the ring

$$
\begin{aligned}
& j_{\varphi}^{\rho}=\frac{c_{s} \zeta_{0}}{L} \int \mathrm{d} \varphi\left[-n_{z}\left(n_{\rho}+\frac{\partial n_{\varphi}}{\partial \varphi}\right)+n_{\varphi} \frac{\partial n_{z}}{\partial \varphi}\right], \\
& j_{\varphi}^{\varphi}=\frac{c_{s} \zeta_{0}}{L} \int \mathrm{d} \varphi\left[n_{z}\left(-n_{\varphi}+\frac{\partial n_{\rho}}{\partial \varphi}\right)-n_{\rho} \frac{\partial n_{z}}{\partial \varphi}\right], \\
& j_{\varphi}^{z}=\frac{c_{s} \zeta_{0}}{L} \int \mathrm{d} \varphi\left[n_{\varphi}\left(n_{\varphi}-\frac{\partial n_{\rho}}{\partial \varphi}\right)+n_{\rho}\left(n_{\rho}+\frac{\partial n_{\varphi}}{\partial \varphi}\right)\right] .
\end{aligned}
$$

Now we can use the following expansion in harmonics:

$$
n_{\mu}(\varphi)=\frac{1}{\sqrt{2 \pi}} \sum_{l} \mathrm{e}^{\mathrm{i} l \varphi} n_{\mu}(l), \quad l=0, \pm 1, \ldots
$$

and, finally, we obtain for the components of spin current

$$
\begin{aligned}
& j_{\varphi}^{\rho}=-\frac{c_{s} \zeta_{0}}{L} \sum_{l}\left[n_{z}(-l) n_{\rho}(l)+\mathrm{i} l n_{z}(-l) n_{\varphi}(l)\right], \\
& j_{\varphi}^{\varphi}=-\frac{c_{s} \zeta_{0}}{L} \sum_{l}\left[(1-\mathrm{i} l) n_{z}(-l) n_{\varphi}(l)-\mathrm{i} l n_{\rho}(-l) n_{z}(l)\right], \\
& j_{\varphi}^{z}=\frac{c_{s} \zeta_{0}}{L} \sum_{l}\left[n_{\varphi}(-l) n_{\varphi}(l)+n_{\rho}(-l) n_{\rho}(l)-2 \mathrm{i} l n_{\varphi}(-l) n_{\rho}(l)\right] .
\end{aligned}
$$

In two particular cases when (i) $n_{\rho}=1, n_{\varphi}=n_{z}=0$ (radial orientation of magnetization at the ring), and (ii) $n_{\varphi}=1, n_{\rho}=n_{z}=0$ (tangent orientation of 
magnetization), we obtain using Eqs. (24) to (26)

$$
j_{\varphi}^{z}=\frac{2 \pi c_{s} \zeta_{0}}{L}, \quad j_{\varphi}^{\rho}=j_{\varphi}^{\varphi}=0 .
$$

It means that there exists a persistent spin current in the ring transferring the $z$-component of magnetization.

It should be emphasized that the spin current (27) is related to the metastable state of magnetization field but not related to spin wave excitations [20]. The contribution of magnons still exists but in this case this contribution is negligibly small because the magnons are weak excitations over the metastable state. Besides, the contribution of magnons is completely vanishing in the limit of $T \rightarrow 0$.

It is known that the persistent spin current in the ring induces a static electric polarization [21, 20], which is associated with the inverse spin Hall effect [14, 22]. Due to this effect the spin current can be observable by measurements of the static electric field near the ring with the persisting current. The electric polarization $\boldsymbol{P}$ is related to the spin current by $P_{i} \simeq \epsilon_{i j \mu} j_{j}^{\mu} / c^{2}$, which allows to estimate the magnitude of induced electric field.

\section{Conclusions}

Using a general approach to the spin current problem we calculated the equilibrium spin current in a mesoscopic ring. This current is related to the inhomogeneous magnetization, which cannot be avoided by any transformations of the ring geometry because of topology of the magnetic excitation in the ring (magnetic vortex). The physical meaning of nondissipative spin current is the transmission of an angular momentum acting locally on the magnetic moment in each point of the ring, which is quite similar to the spin torque mechanism of magnetic moment reversion in the magnetic multilayers.

\section{Acknowledgments}

This work is supported by FCT Grant PTDC/FIS/70843/2006 in Portugal and the Polish Ministry of Science and Higher Education as research projects in years 2006-2009 and 2007-2010.

\section{References}

[1] J.C. Slonczewski, J. Magn. Magn. Mater. 159, L1 (1996); 195, L261 (1999).

[2] L. Berger, Phys. Rev. B 54, 9353 (1996).

[3] A.A. Abrikosov, L.P. Gorkov, I.E. Dzyaloshinski, Methods of Quantum Field Theory in Statistical Physics, Dover, New York 1963.

[4] G.D. Mahan, Many-Particle Physics, Kluwer, New York 2000.

[5] P.M. Levy, Solid State Phys. 47, 367 (1994).

[6] H.E. Camblong, P.M. Levy, S. Zhang, Phys. Rev. B 51, 16052 (1995). 
[7] E.B. Sonin, cond-mat arXiv:0807.2524 (2008).

[8] F. Gross, Relativistic Quantum Mechanics and Field Theory, Wiley, New York 1999, Ch. 13.

[9] A. Crépieux, P. Bruno, Phys. Rev. B 64, 014416 (2001).

[10] N. Nagaosa, J. Phys. Soc. Jpn. 75, 042001 (2006).

[11] J. Inoue, H. Ohno, Science 309, 2004 (2005).

[12] J. Schliemann, Int. J. Mod. Phys. B 20, 1015 (2006).

[13] H.A. Engel, E.I. Rashba, B.I. Halperin, in: Handbook of Magnetism and Advanced Magnetic Materials, Eds. H. Kronmüller, S. Parkin, Vol. 5, Spintronics and Magnetoelectronics, Wiley, New York 2007, p. 1.

[14] J.E. Hirsch, Phys. Rev. Lett. 83, 1834 (1999).

[15] G.A. Prinz, Science 282, 1660 (1998); S.A. Wolf, D.D. Awschalom, R.A. Buhrman, J.M. Daughton, S. von Molnár, M.L. Roukes, A.Y. Chtchelkanova, D.M. Treger, Science 294, 1488 (2001); I. Zutić, J. Fabian, S. Das Sarma, Rev. Mod. Phys. 76, 323 (2004).

[16] J. Shi, P. Zhang, D. Xiao, Q. Niu, Phys. Rev. Lett. 96, 076604 (2006); P. Zhang, Z. Wang, J. Shi, Di Xiao, Q. Niu, Phys. Rev. B 77, 075304 (2008).

[17] A.A. Belavin, A.M. Polyakov, JETP Lett. 22, 245 (1975).

[18] V.L. Pokrovsky, M.V. Feigel'man, A.M. Tsvelick, in: Spin Waves and Magnetic Excitations 2, Eds. A.S. Borovik-Romanov, S.K. Sinha, Elsevier, Amsterdam 1988 , p. 67.

[19] P. Chandra, P. Coleman, A.I. Larkin, J. Phys., Condens. Matter 2, 7933 (1990).

[20] F. Schütz, M. Kollar, P. Kopietz, Phys. Rev. Lett. 91, 017205 (2003); Phys. Rev. $B$ 69, 035313 (2004).

[21] F. Meier, D. Loss, Phys. Rev. Lett. 90, 167204 (2003).

[22] T. Kimura, Y. Otani, T. Sato, S. Takahashi, S. Maekawa, Phys. Rev. Lett. 98, 156601 (2007). 